\title{
Object- and direction-specific interference between manual and mental rotation
}

\author{
Alexander T. SaCk And Michael Lindner \\ Maastricht University, Maastricht, The Netherlands \\ and Johann Wolfgang Goethe University, Frankfurt, Germany \\ AND \\ DAVID E. J. Linden \\ University of Wales, Bangor, Wales \\ and Johann Wolfgang Goethe University, Frankfurt, Germany
}

\begin{abstract}
We conducted a series of psychophysical experiments to investigate the nature and specificity of behavioral interference between mental and manual rotation. Participants were asked to mentally rotate five different types of visual stimuli - hands, faces, tools, cubes, and natural objects - either clockwise or counterclockwise while they simultaneously manually rotated a wheel in the concordant or discordant direction. Our study clearly revealed object-specific interference between manual and mental rotation. In comparison with a neutral baseline condition without any manual rotation, both manual rotation directions generally impaired the mental rotation of cubes. In contrast, the mental rotation of hands was impaired only by discordant manual rotation. This objectand direction-specific interference between manual and mental rotation proved to be independent of task difficulty. We furthermore found an angular distance effect across different stimulus types.
\end{abstract}

The processes of visual imagery - generating, examining, and manipulating visual mental images (Palmer, 1999; Richardson, 1999) — are in many ways parallel to those of visual perception. Striking similarities have been reported not only in subjective accounts, but also in performance measures and brain activation patterns (Kosslyn \& Thompson, 2003). The understanding of visual imagery has important implications for theories of conscious experience. For the cognitive psychologist, visual imagery is of interest mainly because it plays a crucial role in several core cognitive abilities, such as memory (Goldstein, 2002), high-level vision (Kosslyn, 1994; Tarr, 1999), and object recognition (Riesenhuber \& Poggio, 2000). Visual imagery also supports the identification of the shortest route between two locations in spatial orientation (Goldstein, 2002) and is instrumental in abstract reasoning, skill learning, and language comprehension (Kosslyn, Behrmann, \& Jeannerod, 1995).

The quality of visual imagery has been debated since the very beginnings of scientific psychology; at issue specifically is whether visual imagery is based on an abstract code that can also be found in language (Anderson \& Bower, 1973; Pylyshyn, 1973) or on a picture-like representation (James, 1890; Kosslyn, 1980, 1994; Paivio, 1971). An important contribution to this debate has come from the investigation of spatial manipulations of imagined objects. Objects in mental imagery can be manipu- lated much like actual objects, and potential interactions between physical and mental manipulations (Kosslyn, Digirolamo, Thompson, \& Alpert, 1998) might help resolve these theoretical issues. According to Kosslyn (1994), image transformations are not accomplished by a single process, but rather by two types of image transformations. The so-called motion-encoded transformations occur when one activates a representation of an object that was encoded during movement, producing a moving image, whereas motion-added transformations occur when an imagined stationary object is caused to move by the imagery process in a novel way.

Image transformation processes have most widely been investigated using mental rotation paradigms. Mental rotation refers to the ability to imagine objects' changing their orientations in 3-D space. The classical task of visual mental rotation was described by Shepard and Metzler in 1971. In their task, pairs of geometrical figures (3-D objects made out of cubes) were presented to the subjects. In these pairs, one object either was identical to the second or was its mirror image but was rotated at a certain angle. When subjects had to compare these two geometrical figures and judge whether the second object was identical to or the mirror image of the first, their reaction times (RTs) increased linearly as the degree of rotation increased. Subjects reported that they imagined mentally rotating the figure and maintaining in-between positions of the rotated

D. E. J. Linden, d.linden@bangor.ac.uk 
object while comparing it with the target sample. Thus, the larger the angular distance between the two samples, the more intermediate positions of the rotated object had to be imagined and maintained. This paradigm shows that behavioral performance in visual mental imagery is a function of the demand of the task, which can be analogous to that of a corresponding physical task.

Similar effects have been observed when body parts are mentally transformed, albeit with some important differences. RTs for mental rotations of line drawings of hands increase with increases in the rotation angle (Sekiyama, Miyauchi, Imaruoka, Egusa, \& Tashiro, 2000), but, unlike RTs for mental rotations of cubes, they do not always peak at $180^{\circ}$ (Sekiyama, 1982). This can be explained by the specific anatomical constraints of the joints involved in hand movements that make certain orientations particularly awkward. Studies involving spatial judgments of photographs of rotated human bodies confirmed that RTs do not simply align with rotation angle but are influenced by familiarity with particular orientations of human bodies in the physical world (Parsons, 1987). The link between motion imagery and actual movement is also supported by the observation that the pure mental imagination of a limb moving produces an increase in specific limb reflexes similar to the actual movement of that limb (Kosslyn et al., 1995). These results raise questions about potential common neuronal bases for mental and physical rotation and about the differences between transformations of body parts and transformations of other types of visual stimuli.

Wohlschläger and Wohlschläger (1998) showed that concurrent manual rotation interferes with mental rotation of cubes. This behavioral interference between manual and mental rotation was found when the axes of mental rotation and a simultaneous hand movement coincided in space. The authors concluded that concordant rotational directions facilitate mental rotation but discordant directions have an inhibitory effect. This interference effect was found for both hands, although it was less pronounced for the nondominant hand. This interference between rotational hand movement and mental rotation provided strong evidence that mental and object rotations relied on a common process. In a second study, Wohlschläger (2001) demonstrated that the mere planning of a manual rotation that was discordant with the presumed mental rotation resulted in longer RTs, indicating behavioral interference between the planned manual rotation and mental rotation. Several researchers (Wexler, Kosslyn, \& Berthoz, 1998; Wohlschläger, 2001) have suggested that mental rotation should be described as an imagined covert action, rather than as pure visuospatial imagery, and that the observed interference between manual and mental rotation is the result of both types of rotation's relying on the same system for action planning.

The common neural basis of imagined and physical rotation has been investigated with functional magnetic resonance imaging (fMRI). Carpenter, Just, Keller, Eddy, and Thulborn (1999) correlated the increments of RTs during mental rotation of cubes with changes in regional cerebral activation. With higher angular disparity, activation increased bilaterally in the parietal lobes but not in the temporal lobe. This discrepancy indicates that the parietal cortex plays a central role in the visuospatial transformations of mental rotation (Formisano et al., 2002; Goebel, Linden, Lanfermann, Zanella, \& Singer, 1998; Sack et al., 2002), whereas the ventral (temporal) pathway, which is essential for identifying a figure, does not specifically support this operation. Activity in the motor areas of the frontal lobe was significantly higher during the mental rotation paradigm than it was in a control condition involving a high number of saccadic eye movements. This difference suggests that "the so-called motor areas are not simply involved in motor planning and execution" (Carpenter et al., 1999, p. 18) but play a crucial role in the computation of imagined motion of objects as well. These findings indicate that Shepard-and-Metzler-like tasks are indeed performed by mentally rotating an object as if it were moving through the intermediate positions along a trajectory, as would occur if the object were physically rotated (Kosslyn et al., 1998).

fMRI has also been applied to the study of the neural basis of mental transformation of body parts. Several imaging studies revealed the involvement of premotor areas during the mental rotation of hand stimuli (Bonda, Petrides, Frey, \& Evans, 1995; Parsons et al., 1995) and geometric objects (Cohen et al., 1996; Lamm, Windischberger, Leodolter, Moser, \& Bauer, 2001; Richter et al., 2000). In addition to activity in the premotor cortex and the supplementary motor area, Kosslyn et al. (1998) even found activation in the primary motor cortex (PMC) when subjects imagined rotating hands, the functional relevance of which was confirmed by the transcranial magnetic stimulation study of Ganis, Keenan, Kosslyn, and PascualLeone (2000). PMC activity has also been reported occasionally for mental rotation of geometric objects, but this might have been confounded by the buttonpress response required for the task (Richter et al., 2000). Activation of the hand and foot areas of the PMC was also found when participants imagined moving those body parts (Ehrsson, Geyer, \& Naito, 2003). Georgopoulos and Pellizzer (1995) provided evidence from single-cell recordings in the motor cortex of monkeys that direction-specific cells were active along an imaginary motion trace.

The results of Kosslyn et al. (1995), in particular, provide the functional imaging background to the present study. Kosslyn et al. (1995) used positron emission tomography (PET) to monitor regional cerebral blood flow while participants mentally rotated hand or cube stimuli. Substantial activation in motor areas, including PMC, was found for the hands task. In contrast, when Kosslyn et al. (1995) examined the areas that were activated in the cube conditions, they found activation in parietal regions but not in frontal motor regions. The results suggest at least two different ways to perform mental rotation, only one of which involves the cortical motor system (Kosslyn et al., 1998). These imaging results are far from trivial, because the notion that mentally rotating objects involves having them travel through trajectories does not imply that the observer's own motor system must be manipulating the objects. One could imagine that objects are rotated by someone else or are shifted by physical forces. Indeed, it 
is possible that the imagined method used is tailored to the specific task or to the specific stimuli. If so, we may find differences in demands on motor processing when hands versus Shepard-Metzler objects are rotated. Although the imaging literature thus points to an interesting dissociation between mental rotations of hands (and potentially other body parts) and other objects, no previous behavioral study has directly compared the potential facilitating and inhibitory effects of hand rotation on the mental transformation of body and nonbody stimuli.

The goal of the present study was to systematically investigate the stimulus-dependent influence of directionspecific manual rotation on performance in a mental rotation task. Participants had to perform a mental rotation task in which images of geometric objects (Shepard-Metzler figures), natural objects (bundles of carrots), tools, or body parts (pictures of hands or faces) had to be mentally rotated in clockwise and counterclockwise directions. While performing this mental rotation, participants were asked to manually rotate a custom-made device. This manual rotation condition required counterclockwise, clockwise, or no manual rotation. Because the imaging literature indicates that the motor system is involved in at least some types of mental rotation, an interaction of the motor activity evoked by manual rotation with mental rotation was expected.

\section{EXPERIMENT 1 \\ Stimulus-Specific Interference Between Manual and Mental Rotation}

We investigated the hypothesis that stimulus- and direction-specific interference between manual and mental rotation exists by performing a series of behavioral experiments that successively ruled out a number of possible alternative explanations for our findings. In the first experiment, we investigated whether manual rotation with the left hand had a stimulus-specific behavioral effect on a mental rotation task when manual and mental rotation had to be performed simultaneously in either a concordant or a discordant direction. We explicitly distinguished between different stimulus categories, which we expected to be differentially vulnerable to such an interference effect.

\section{Method}

Participants. The 40 right-handed participants ( 20 females and 20 males; mean age $=29.73$ years, $S D=9.02$, range $=19-59)$ were psychology students from Frankfurt University who received course credit for their participation. None of the participants reported any health problems, and all had normal or corrected-to-normal vision. Participants were unaware of the purposes and predictions of the experiment until after test completion.

Stimuli. All stimuli were created using Adobe Photoshop with a 96-dpi resolution. Three different stimulus types were used: abstract figures, non-body-part figures, and hand figures. The abstract figures (cube figures) were constructed by juxtaposing 12 cubes in all three dimensions, similar to Shepard and Metzler's (1971) design. For the non-body-part figures, computer-painted bundles of carrots were used; 12 different carrots and two different configurations of leaves were painted and randomly combined to create 24 different bundles, each containing 5 carrots. The hand figures consisted of 24 digitized photographs of a female's left hand. For each hand figure, the arrangement of the fingers was altered by having the fingers and thumb bent in different ways. To create stimulus pairs, two identical figures were placed on a white background $(800 \times 500$ pixels $)$. The figure on the right was either a copy or a mirror image of the figure on the left and was rotated by $40^{\circ}, 80^{\circ}$, or $120^{\circ}$ (clockwise or counterclockwise in the screen plane). See Figure 1 for examples of the stimuli used. Stimuli were designed in such a way that the rotational angles worked for only one direction of mental rotation; that is, in the clockwise condition, a $40^{\circ}$ rotational angle was defined by $40^{\circ}$ rotation of the right stimulus in the clockwise direction, whereas in the counterclockwise condition, a $40^{\circ}$ rotational angle was defined by $40^{\circ}$ rotation of the right stimulus in the counterclockwise direction. In this way, 48 different stimulus pairs were created for each stimulus type ( 2 stimulus rotation directions $\times 3$ stimulus rotation angles $\times 2$ identical/mirror images $\times 4$ repetitions). The basic figures were distributed to the manual rotation condition, stimulus rotation angle, and identical/mirror image conditions in a randomized way.

Apparatus and Procedure. Participants were seated in front of a 14-in. LCD screen $(1,024 \times 768$ pixels $)$ on a height-adjustable stand. Each participant's head was mounted on a chin-forehead rest, ensuring that the eyes were centered in vertical and horizontal directions at a 35-cm distance from the screen. An AMD Athlon 2000+ Notebook controlled the experiment; stimuli were displayed using Presentation (Neurobehavioral Systems, Version 0.71, www.neurobs.com).

On each trial, two stimuli were presented at a visual angle of about $9.25^{\circ}$ left and right of the screen's center. Half of the participants were asked to mentally rotate the left stimulus figure clockwise, and the other half were instructed to mentally rotate the left stimulus figure counterclockwise, until they identified the stimulus as either identical to (left buttonpress) or the mirror image of (right buttonpress) the right stimulus. Prior to each block, participants were always told in which direction to mentally rotate the stimuli (counterclockwise or clockwise). Stimuli were designed in such a way that the rotational angles always corresponded to the instructions given to the participants, so that following the instructions led to the shortest rotation path. The stimuli were presented until the participant responded or for a maximum of $10 \mathrm{sec}$ (interstimulus interval $=4 \mathrm{sec}$ ).

While mentally rotating the stimuli, participants also had to manually rotate a custom-made manual rotation device (MRD). The MRD consisted of a wooden box $(21 \mathrm{~cm}$ high $\times 30 \mathrm{~cm}$ wide $\times 21 \mathrm{~cm}$ deep) with a turnable wooden wheel $(10 \mathrm{~cm}$ in diameter) that did not provide resistance. The MRD was placed on a table in front of the participant in such a way that the turnable wheel faced the participant's hand when it rested on the table. Depending on the experimental condition, participants were asked to manually turn the rotation device with either their left or their right hand as instructed - to the left, to the right, or not at all. The participants performed this manual rotation during the presentation of the visual stimuli and stopped rotating when the stimulus disappeared - that is, after they had made their response. After responding, participants unclasped the wheel and rotated their wrist back into the neutral start position in order to be ready for the next trial. The experimenter ascertained by observation that participants rotated continuously in the given direction during stimulus presentation without any interruptions or adjustments. This procedure was practiced before the actual experiment.

The experiment followed a $2 \times 3 \times 3 \times 3$ mixed four-factor design, with mental rotation direction (clockwise or counterclockwise) as a between-participants factor with two levels and manual rotation direction (concordant, none, or discordant), stimulus rotation angle $\left(40^{\circ}, 80^{\circ}\right.$, or $\left.120^{\circ}\right)$ and stimulus type (cube, carrots, or hand figures) as within-participants factors with three levels each.

Each participant took part in three experimental blocks, one for each stimulus type. The order of the blocks was counterbalanced across participants. The blocks were subdivided into three sessions corresponding to the three hand movement conditions. Each session contained 24 trials and was preceded by an instruction for the rotation direction of the left hand. Throughout the experiment, the experimenter sat behind the participant to control whether the participant turned the wheel in the correct direction and at the correct speed. 


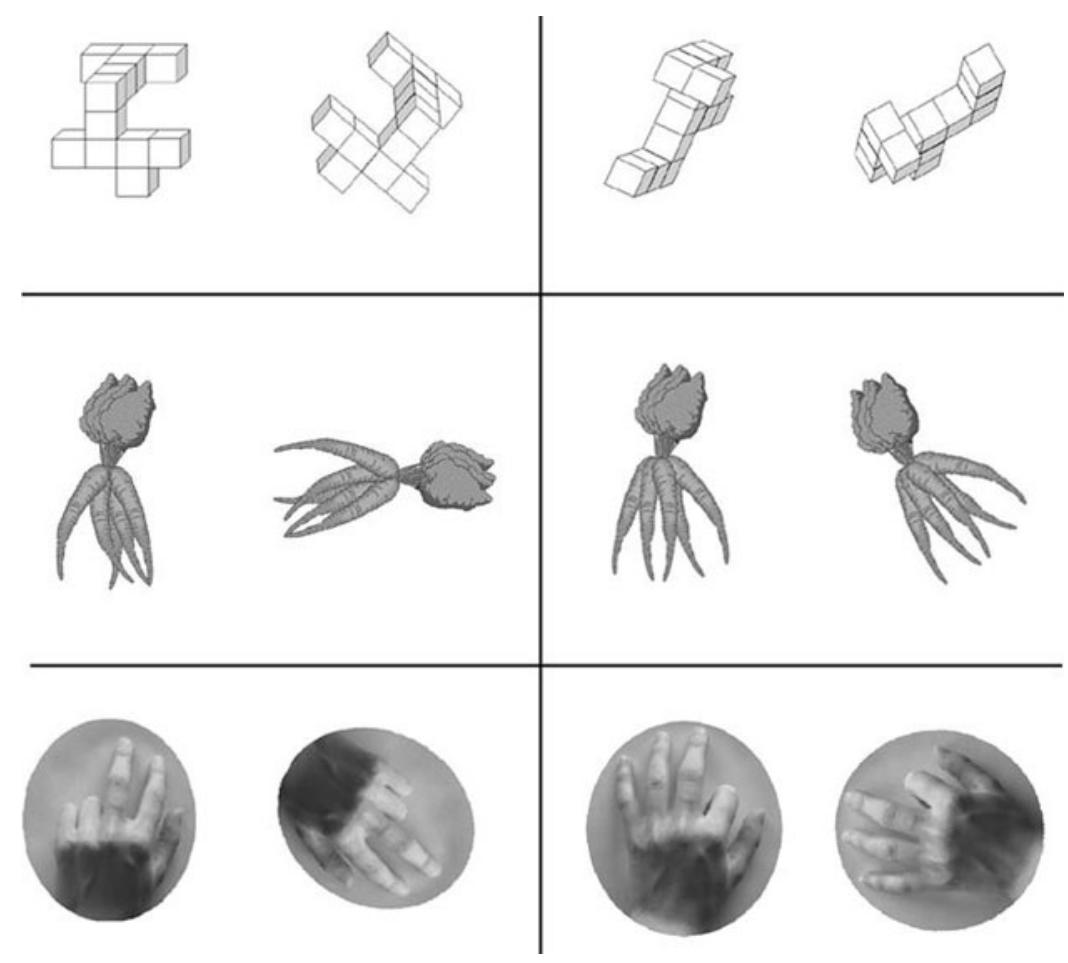

Figure 1. Illustrations of the identical and mirror image conditions in the cube figures (top), carrots (middle), and hands (bottom) in the clockwise and counterclockwise (images on the left and right sides, respectively) conditions.

Before the experiment, all participants took part in a training session consisting of three runs, which contained all three hand movement conditions and stimulus types. They were instructed and trained to rotate the MRD at a speed of approximately $90^{\circ}$ per second.

Data analysis. Prior to the inference statistical testing, participants with an error rate of $20 \%$ or higher were identified and their data were excluded from further analyses. This resulted in the elimination of 4 participants. For the RT data, statistical outliers, defined as participants whose RTs were more than two standard deviations from the mean, were identified and removed. Only trials with correct responses were considered and averaged for the different experimental conditions. The RT data of the correct responses were further tested for normal distribution and variance homogeneity. These tests revealed that the RT data were skewed to the right. In order to obtain a normal distribution, the entire data set underwent a logarithmic transformation. This ensured the suitability of the RT data for parametric statistical testing. Before entering the data into the full-factorial ANOVA, we confirmed that there was no significant difference between identical and mirror image trials. Tests of significance were performed using a four-factor repeated measures ANOVA with (1) rotational angle (three levels), (2) stimulus type (three levels), and (3) manual rotation direction (three levels) as the three repeated measures factors and mental rotation direction (two levels) as the one between-participants factor. On the basis of the results of this full-factorial ANOVA, several simple post hoc contrast analyses were performed for each condition.

\section{Results}

Stimulus type. The average RT required to perform the mental rotation differed significantly among the three stimulus types (cubes, carrots, and hand stimuli). Stimulus type showed a significant main effect within the fourfactor ANOVA $[F(2,35)=72.64, p<.001]$. Post hoc simple contrast analyses revealed that the RTs for the cube figures $(M=3,665 \mathrm{msec}, S D=845)$ were significantly higher $[F(1,35)=32.33, p<.001]$ than those for the carrot figures $(M=3,027 \mathrm{msec}, S D=896)$, which in turn were significantly higher $[F(1,35)=44.63, p<$ $.001]$ than the RTs for the hand figures $(M=2,202 \mathrm{msec}$, $S D=725)$.

Stimulus rotation angle. As Figure 2 shows, RTs systematically increased with increasing angular disparities, from $2,696 \mathrm{msec}\left(40^{\circ}\right.$ angle) to $2,961 \mathrm{msec}\left(80^{\circ}\right.$ angle) to $3,238 \mathrm{msec}\left(120^{\circ}\right.$ angle). The rotation angle of the stimulus showed a significant main effect within the four-factor ANOVA $[F(2,35)=76.82, p<.001]$. Post hoc simple contrast analyses revealed that the RTs for the $120^{\circ}$-angle condition were significantly higher $[F(1,35)=64.74, p<$ $.001]$ than those for the $80^{\circ}$-angle condition, which in turn were significantly higher $[F(1,35)=72.59, p<.001]$ than the RTs for the $40^{\circ}$-angle condition. Moreover, this angle distance effect was also found when analyzing separately for the two mental rotation directions [clockwise, $F(2,17)=61.15, p<.001$; counterclockwise, $F(2,17)=$ $52.26, p<.001]$ and for the three different manual rotation conditions [concordant, $F(2,35)=52.06, p<.001$; discordant, $F(2,35)=59.24, p<.001$; none, $F(2,35)=$ $68.50, p<.001]$. This clearly reveals a systematic relationship between the required angle of mental rotation and the respective RT required to perform the mental rotation, and it provides empirical support for the theory that participants did mentally rotate the respective object in the instructed direction. Moreover, the fact that the angular distance effect was not modulated by the direction of man- 


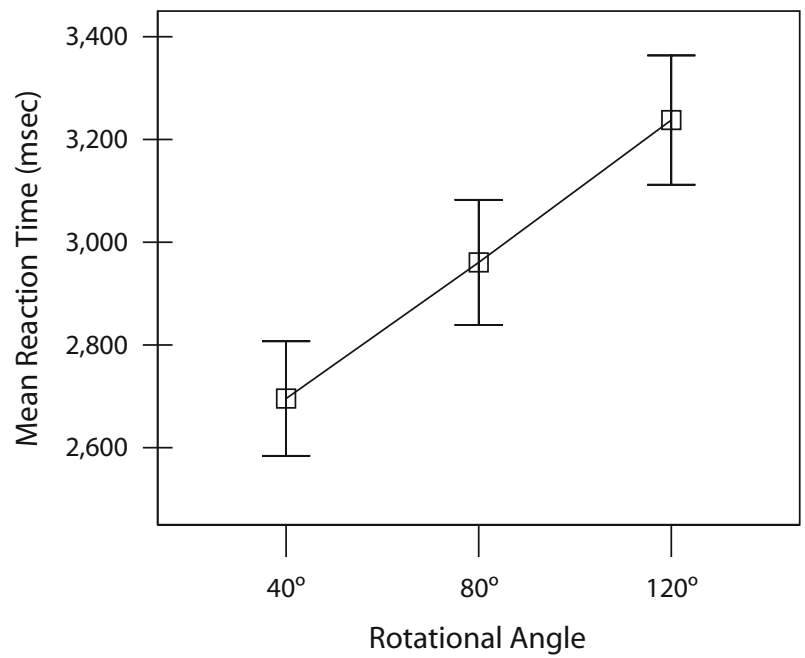

Figure 2. Reaction times for the three rotation angles $\left(40^{\circ}, 80^{\circ}\right.$, and $120^{\circ}$ ) across all stimulus types.

ual rotation [no interaction between angular disparity and manual rotation condition; $F(4,35)=1.33, p=.27]$ indicates that the interference effect of manual on mental rotation, described in the following section, is brought about by a general shift in baseline performance, rather than by a systematic change in the rate of mental rotation.

Manual rotation effect. The four-factor ANOVA not only showed a significant main effect of the manual rotation direction $[F(2,35)=121.40, p<.001]$, but moreover revealed a significant interaction between manual rotation direction and stimulus type $[F(4,35)=3.67, p=.007]$. Hence, the RTs for the three hand movement conditions in relation to the mental rotation direction (concordant direction, no hand movement [neutral], and discordant direction) were compared for each stimulus type separately.

For the cube figures, the fastest RTs were found in the no hand movement condition $(M=3,530 \mathrm{msec}, S D=$ 917; see Figure 3A). In contrast, both of the manual rotation conditions - concordant direction $(M=3,667 \mathrm{msec}$, $S D=991)$ and discordant direction $(M=3,799 \mathrm{msec}$,
$S D=891)$-resulted in an increase in RTs, and the discordant manual rotation showed the slowest RTs in comparison with the RTs of the other two conditions. A post hoc simple contrast analysis revealed that only the difference between the no hand movement and the discordant manual rotation direction was statistically significant $[F(1,35)=7.92, p=.01]$, whereas no significant difference was found between no hand movement and the concordant manual rotation direction $[F(1,35)=1.22, p=$ .28] or between the concordant and discordant manual rotation directions $[F(1,35)=1.12, p=.30]$.

As Figure $3 \mathrm{~B}$ shows, the RTs for the carrot figures showed a descriptive improvement of RTs for the concordant manual rotation direction $(M=2,900 \mathrm{msec}, S D=$ $859)$, as compared with the no hand movement condition $(M=3,092 \mathrm{msec}, S D=1,093)$ and the discordant manual rotation condition $(M=3,089 \mathrm{msec}, S D=972)$, but the main effect of manual rotation direction was not significant $[F(2,35)=1.08, p=.34]$.

For the hand figures, the RTs were descriptively fastest for the concordant manual rotation direction $(M=$ $2,117 \mathrm{msec}, S D=686)$, followed by the no hand movement condition $(M=2,121 \mathrm{msec}, S D=851)$ and the discordant manual rotation direction $(M=2,356 \mathrm{msec}$, $S D=817$; see Figure 3C). A post hoc simple contrast analysis revealed that only the discordant manual rotation direction differed significantly, as compared with no hand movement $[F(1,35)=5.96, p=.02]$ and concordant manual rotation direction $[F(1,35)=6.07, p=.02]$. The contrast between concordant manual rotation direction and no hand movement failed to reach significance $[F(1,35)=0.01, p=.94]$.

\section{Summary}

Experiment 1 demonstrated that performing manual rotation during a mental rotation task has an effect on performance that can be measured by the RT required for mental rotation. Importantly, we found a statistical interaction between manual rotation and stimulus type that suggests clear differences among the three stimulus types in terms of the degree of interference between man-
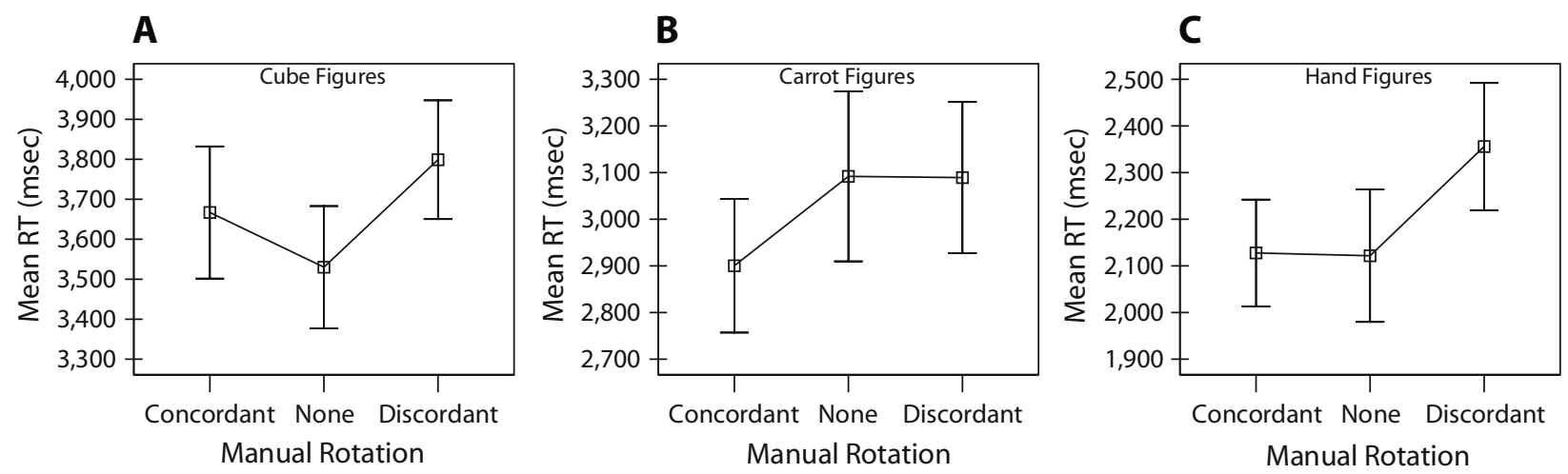

Figure 3. Mean reaction times (RTs) for the manual rotation direction in relation to the mental rotation direction (concordant direction, no hand movement, and discordant direction) for the three stimulus types: cube, carrot, and hand figures. Error bars represent standard errors of the means. 
ual and mental rotation that they cause. Although mental rotation for the cube figures was significantly impaired during discordant manual rotation as compared with no manual rotation, there was no significant difference between the concordant and the discordant manual rotations. In contrast, the hand stimuli were affected by the manual rotation direction in a more specific way. These stimuli were unique in revealing both impaired performance for the discordant manual rotation as compared with no manual rotation and a significant difference in performance between the concordant and discordant manual rotation directions. In the context of this stimulus-type specificity, the mental rotation of hand stimuli showed a clear direction-specific vulnerability to the interfering effect of the manual rotation in comparison with the carrots and cube stimuli. However, this experiment did not reveal a significant improvement during concordant manual rotation in comparison with a mere mental rotation condition without any hand movement.

\section{EXPERIMENT 2 Manual Rotation With Left Versus Right Hand}

In order to investigate whether the hand used for rotation affects these effects, we conducted a second experiment in which participants used either their right or their left hand for the rotation.

\section{Method}

Participants. The 20 right-handed participants ( 10 females and 10 males; mean age $=25.50$ years, $S D=3.30$, range $=21-33)$ were psychology students at Frankfurt University who received course credit for their participation. None of the participants reported any health problems, and all had normal or corrected-to-normal vision. The participants were unaware of the purposes and predictions of the experiment until after test completion.

Stimuli. The 12 basic cube and hand figures from the first experiment were copied and mirrored along the vertical axis. Together with the 12 original cube and hand figures, these mirror copies formed the 24 new basic cube and hand figures for the new stimuli. As a result, we obtained 48 new pairs of cube figures and 48 new pairs of hand figures, which had the same qualities as the pairs of stimuli used in Experiment 1 (see Figure 1).

Apparatus and Procedure. The apparatus and procedure were similar to those used in Experiment 1. On half of the trials, participants had to perform the manual rotation with their right hands, and on the other half, they used use their left hands. To reduce the number of trials, some factor levels were dropped. Participants were presented with only two stimulus types (hand and cube figures); they were instructed to manually rotate to either the left or the right, and the mental rotation was performed in the clockwise direction only.

These modifications resulted in a $2 \times 2 \times 3 \times 2$ repeated measures factorial design with hand (left vs. right), manual rotation direction (concordant vs. discordant), stimulus rotation angle $\left(40^{\circ} \mathrm{vs.}\right.$ $80^{\circ}$ vs. $120^{\circ}$ ), and stimulus type (cube vs. hand figures) as withinparticipants factors.

Data analysis. Prior to the inference statistical testing, participants with an error rate of $20 \%$ or higher were identified and excluded from further analyses. This resulted in the elimination of 1 participant. For the RT data, statistical outliers, defined as participants whose results differed more than two standard deviations from the mean, were identified and removed. Only trials with correct responses were considered and averaged for the different experimental conditions. The entire data set underwent a logarithmic transformation and was tested for normal distribution and variance homogeneity. These tests revealed the suitability of the RT data for parametric statistical testing. Tests of significance were performed using a four-factor repeated measures ANOVA with (1) rotational angle (three levels), (2) stimulus type (two levels), (3) manual rotation direction (two levels), and (4) hand (two levels) as the four repeated measures factors. On the basis of the results of this full-factorial ANOVA, several simple post hoc contrast analyses were performed for each condition.

\section{Results}

Stimulus type. The average RT required to perform the mental rotation differed significantly between the two stimulus types. The RTs for the cube figures $(M=3,334 \mathrm{msec}$, $S D=941)$ were significantly higher $[F(1,18)=41.03$, $p<.001]$ than those for the hand figures $(M=2,181 \mathrm{msec}$, $S D=512)$.

Stimulus rotation angle. The angular distance effect as described in Experiment 1 was replicated in this experiment. RTs increased from the $40^{\circ}$ angle $(M=2,474 \mathrm{msec}$, $S D=562.11)$ to $80^{\circ}(M=2,798 \mathrm{msec}, S D=620.74)$ to $120^{\circ}(M=3,001 \mathrm{msec}, S D=783.86)$. The rotation angle of the stimulus showed a significant main effect within the four-factor ANOVA $[F(2,18)=48.14, p<.001]$, and post hoc simple contrast analyses revealed that RTs for the $120^{\circ}$-angle condition were significantly higher than those for the $80^{\circ}$-angle condition $[F(1,18)=12.98, p=$ $.002]$, which in turn were significantly higher than the RTs for the $40^{\circ}$-angle condition $[F(1,18)=66.64, p<.001]$. Moreover, this angular distance effect was also found when analyzing separately for the two stimulus types [cube figures, $F(2,18)=24.86, p<.001$; hand figures, $F(2,18)=45.04, p<.001]$ and the hand used to perform the manual rotation [left hand, $F(2,18)=37.56, p<.001$; right hand, $F(2,18)=29.08, p<.001]$.

Manual rotation effect. Although the main effect of the manual rotation factor failed to reach significance $[F(1,18)=3.61, p=.07]$, the four-factor ANOVA revealed a significant interaction between manual rotation and stimulus type $[F(2,18)=4.55, p=.04]$. Although the RTs for the cube stimuli during concordant manual rotation $(M=3,345 \mathrm{msec}, S D=934)$ were almost identical to the RTs during discordant manual rotation ( $M=$ 3,324 msec, $S D=982$; see Figure 4A), the RTs for the hand stimuli increased during the discordant manual rotation $(M=2,349 \mathrm{msec}, S D=597)$, as compared with RTs during the concordant manual rotation $(M=2,013 \mathrm{msec}$, $S D=604$; see Figure 4B). A post hoc simple contrast analysis revealed that the two manual rotation directions (concordant and discordant with the mental rotation direction) showed a significant difference in RT only for the hand stimuli $[F(1,18)=4.48, p=.048]$, whereas for the cube stimuli, no manual rotation effect was revealed $[F(1,18)=0.22, p=.64]$.

The stimulus specificity of the interference between manual and mental rotation is thus statistically supported by a significant interaction between manual rotation and stimulus type $[F(2,18)=4.55, p=.04]$ within the fourfactor ANOVA for repeated measures and by the significant post hoc contrast between concordant and discordant rotation for hand but not cube figures. 

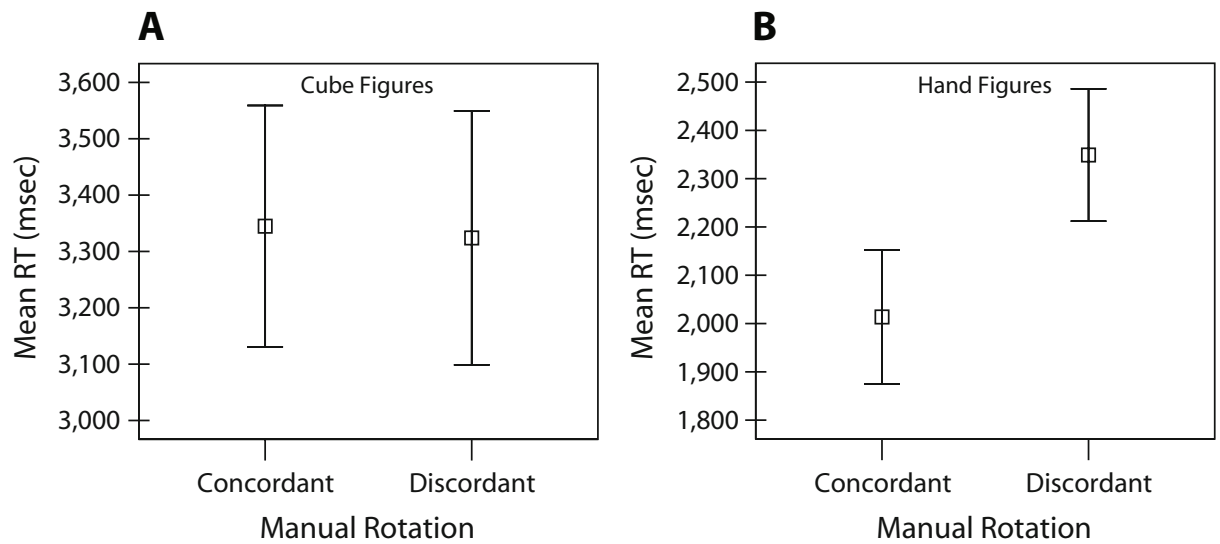

Figure 4. Reaction times (RTs) for the cube and hand figures for the two hand movement conditions (concordant and discordant) averaged across the left and right hands. Error bars represent standard errors of the means.

Hand effect. In accordance with the assumption that the hand with which the manual rotation is performed has a significant impact on the manual rotation interference effect, we found a significant second-order interaction effect among manual rotation direction, stimulus type, and hand condition $[F(1,18)=4.72, p=.04]$. Hence, the interference effect of manual rotation seems not only to be direction and stimulus specific, but also to depend on the hand with which the manual rotation is performed. During the mental rotation of hand stimuli, manual rotation in the discordant direction with the right hand had a stronger impairing effect than did manual rotation in the discordant direction with the left hand (see Figure 5A). For the cube stimuli, however, no such influence of the hand with which the manual rotation had to be performed was observed (see Figure 5B).

\section{Summary}

This second experiment clearly replicated the main finding of Experiment 1. Again we showed that the quality and quantity of the interference between manual and mental rotation depend on the type of stimulus. Although for the cube figures no significant difference between concordant and discordant manual rotation during mental rotation was found, the hand stimuli showed a significant difference between both manual rotation directions, revealing a direction-specific interference between manual and mental rotation. Within this interference effect, a discordant manual rotation, as compared with a concordant manual rotation, led to significantly higher RTs in the mental rotation of hand stimuli. Interestingly, this effect also depended on which hand was used for the manual rotation. As the interaction between manual rotation, stimulus type, and hand revealed, manually rotating with the right hand affected the mental rotation of hand stimuli more strongly.

\section{EXPERIMENT 3}

\section{Stimulus Difficulty and Speed of Rotation}

Both Experiments 1 and 2 showed a significant difference in stimulus difficulty across stimulus types. Hence,
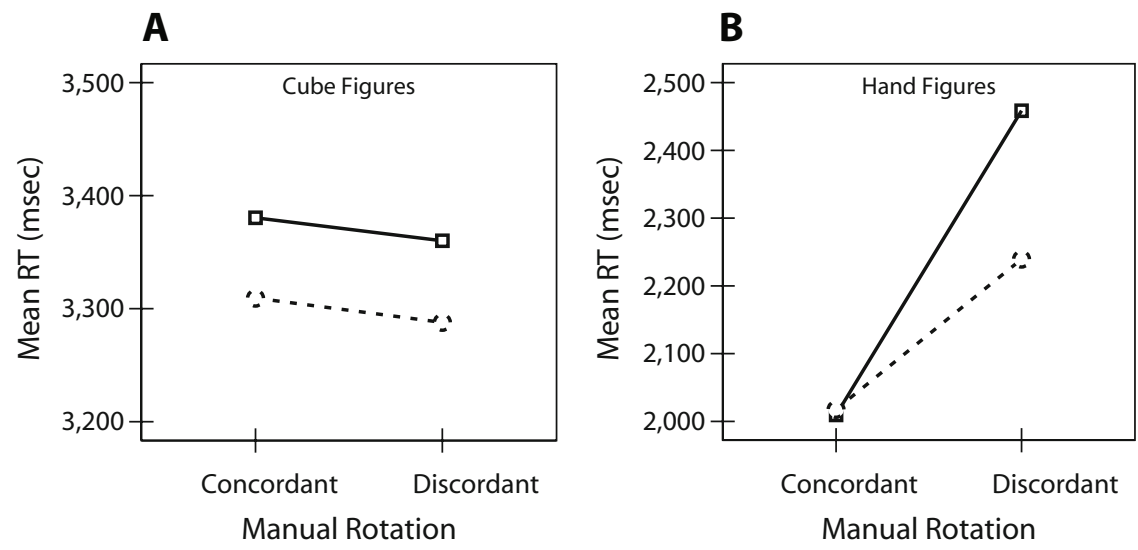

Figure 5. Interaction of the manual rotation direction and the hand (left or right) that was used for the rotation (dotted line = left hand; solid line = right hand), shown separately for the cube figures and the hand figures. 
it cannot be ruled out that the described stimulus-specific interference effect between manual and mental rotation was caused by the differences in task difficulty among the stimuli. In a third experiment, we tried to replicate our main finding with reversed difficulty levels.

\section{Method}

Participants. The 16 right-handed participants ( 8 females and 8 males; mean age $=31.75$ years, $S D=12.19$, range $=22-62$ ) were psychology students at Frankfurt University who received course credit for their participation. None of the participants reported any health problems, and all had normal or corrected-to-normal vision. Participants were unaware of the purposes and predictions of the experiment until after test completion.

Stimuli. The stimuli were comparable to the cube and hand stimuli used in Experiment 1 but were designed to produce reversed difficulties. The new cube figures were created by juxtaposing 6 cubes instead of 12. For the new hand figures, we tried to use nearly symmetrical forms of hands to make their mental rotation more difficult. (For examples of the stimuli used, see Figure 6.)

Apparatus and Procedure. The apparatus and procedure were identical to those used in Experiment 1. However, unlike in Experiment 1 , only cube and hand figures had to be mentally rotated, and the speed of manual rotation with the left hand was now $45^{\circ}$ per second.

The overall structure of the third experiment was a $3 \times 3 \times 2 \times 2$ factorial design, with manual rotation direction (concordant vs. none vs. discordant), rotation of the stimuli $\left(40^{\circ}\right.$ vs. $80^{\circ}$ vs. $\left.120^{\circ}\right)$, stimulus type (cube vs. hand figures), and mental rotation direction (clockwise vs. counterclockwise) as within-participants factors.

Data analysis. Prior to the inference statistical testing, participants with an error rate of $20 \%$ or higher were identified and their data were excluded from further analyses. This resulted in the elimination of 1 participant. For the RT data, statistical outliers, defined as participants whose results differed more than two standard deviations from the mean, were identified and removed. Only trials with correct responses were considered and averaged for the different experimental conditions. The entire data set underwent a logarithmic transformation and was tested for normal distribution and variance homogeneity. These tests revealed the suitability of the RT data for parametric statistical testing. Tests of significance were performed using a four-factor repeated measures ANOVA with (1) rotational angle (three levels), (2) manual rotation direction (three levels), (3) stimulus type (two levels), and (4) mental rotation direction (two levels) as the four repeated measures factors. On the basis of this full-factorial ANOVA, several simple post hoc contrast analyses were performed for each condition.

\section{Results}

The four-factor ANOVA revealed significant main effects for stimulus type $[F(1,14)=5.70, p=.03]$, stimulus rotation angle $[F(2,14)=28.87, p<.001$, and manual rotation direction $[F(2,14)=6.14, p=.01]$. Furthermore, we found significant first-order interactions between manual rotation direction and stimulus type $[F(2,14)=$ $6.17, p=.01]$ and manual and mental rotation $[F(2,14)=$ $4.74, p=.02]$ and a significant second-order interaction between manual rotation direction, stimulus type, and mental rotation direction $[F(2,14)=4.99, p=.02]$. All other possible main, first-order, or second-order interaction effects were nonsignificant.

Stimulus difficulty. As in Experiment 1, the stimulus types differed significantly in their difficulty as measured by the RT required for mental rotation. However, as was intended during the creation of the new stimuli, and in contrast to Experiment 1, the cube figures had the faster RTs $(M=1,758 \mathrm{msec}, S D=714)$ in comparison with the hand figures $(M=2,197 \mathrm{msec}, S D=913)$. Statistically, this difference in RT between both stimulus types was also significant $[F(1,14)=5.70, p=.03]$. Hence, the difficulty difference between both stimulus types was reversed, as compared with Experiment 1.

Angular distance effect. Also in this third experiment, an increase in RTs was found as a function of the degree of the rotation angle. The RTs systematically increased from the $40^{\circ}$-angle condition $(M=1,993 \mathrm{msec}, S D=$ $473)$ to the $80^{\circ}$-angle condition $(M=2,217 \mathrm{msec}, S D=$ $641)$ to the $120^{\circ}$-angle condition $(M=2,329 \mathrm{msec}, S D=$ 635). A highly significant angle distance effect was found $[F(2,14)=28.87, p<.001]$, and post hoc simple contrast analyses revealed that the RTs for the $120^{\circ}$-angle condition were significantly higher than those for the $80^{\circ}$-angle condition $[F(1,14)=16.67, p=.001]$, which in turn were sig-

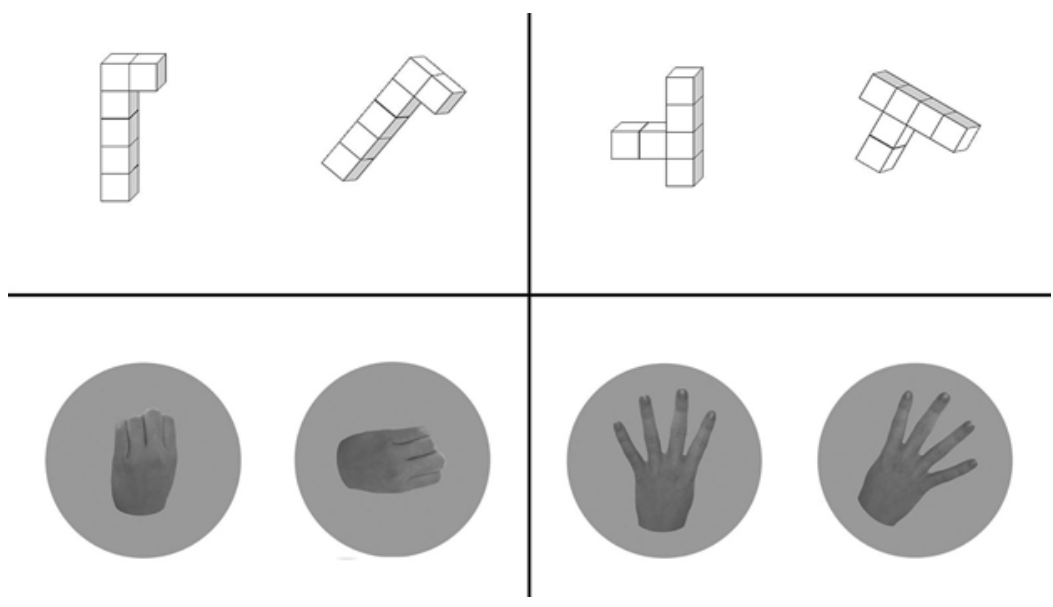

Figure 6. Illustrations of the identical and mirror image conditions (on the left and right, respectively) in the new cube figures (top) and the new hand figures (bottom) in the clockwise condition. 

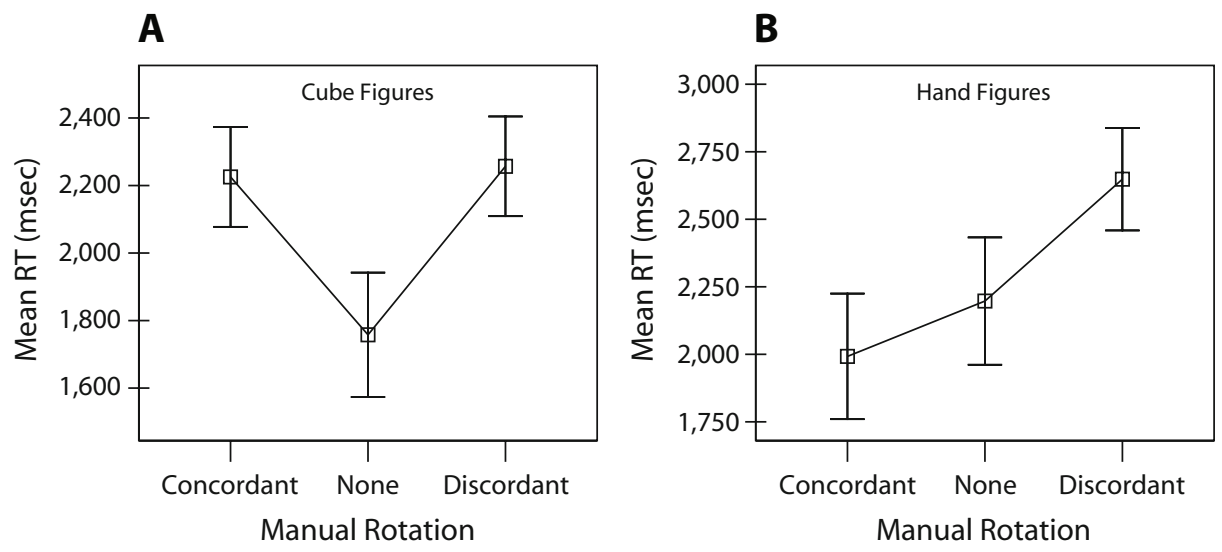

Figure 7. Mean reaction times (RTs) for the manual rotation direction in relation to the mental rotation direction (concordant direction, no hand movement, and discordant direction) for the two stimulus types: cube and hand figures. Error bars represent standard errors of the means.

nificantly higher than the RTs for the $40^{\circ}$-angle condition $[F(1,14)=17.82, p=.001]$. Moreover, this angle distance effect was also found when analyzing the two stimulus types separately [cube figures, $F(2,14)=28.60, p<.001$; hand figures, $F(2,14)=15.18, p<.001]$ and the two mental rotation directions [clockwise, $F(2,14)=27.06, p<$ .001 ; counterclockwise, $F(2,14)=17.31, p<.001]$.

Manual rotation effect. The four-factor ANOVA not only showed a significant main effect of the manual rotation direction $[F(2,14)=6.14, p=.01]$, but moreover revealed a significant interaction between manual rotation direction and stimulus type $[F(2,14)=6.17, p=.01]$. Hence, as in Experiment 1, the interference effect of manual rotation in relation to the mental rotation direction was analyzed separately for the two stimulus types.

As Figure 7A illustrates, for the cube figures, the RTs were again fastest in the no hand movement condition ( $M=1,758 \mathrm{msec}, S D=714)$, whereas both manual rotation directions (concordant, $M=2,226 \mathrm{msec}, S D=572$; discordant, $M=2,257 \mathrm{msec}, S D=572$ ) showed a clear manual interference effect. A post hoc simple contrast analysis revealed that the no hand movement condition significantly differed from the concordant manual rotation direction $[F(1,14)=8.46, p=.01]$ and the discordant manual rotation direction $[F(1,14)=11.25, p=.01]$. However, the contrast between concordant and discordant manual rotation direction failed to reach significance $[F(1,14)=0.18, p=.68]$.

As Figure 7B illustrates, for the hand stimuli, the fastest RTs were found in the concordant manual rotation direction $(M=1,992 \mathrm{msec}, S D=901)$, followed by those for the no hand movement condition $(M=2,197 \mathrm{msec}$, $S D=913)$ and finally, by those for the discordant manual rotation direction $(M=2,649 \mathrm{msec}, S D=735)$. The post hoc contrast analyses revealed that there was a significant difference between the two manual rotation directions $[F(1,14)=6.82, p=.02]$. However, although the hand stimuli also revealed a significant difference between discordant manual rotation and no hand movement $[F(1,14)=6.45, p=.02]$, the difference between no hand movement and concordant manual rotation was not significant $[F(1,14)=1.50, p=.24]$.

Like the analyses derived from Experiment 1, these analyses suggest that the interference effect between manual and mental rotation differed between the two stimulus types. This is again statistically supported by the significant interaction between manual rotation and stimulus type $[F(2,14)=6.17, p=.01]$ within the four-factor ANOVA for repeated measures (see Figures $8 \mathrm{~A}$ and $8 \mathrm{~B}$ ).

\section{Summary}

In this experiment, the difficulty level of the cube and hand stimuli was reversed, as compared with the preceding experiments. We still replicated our main finding, with a significant difference between concordant and discordant manual rotation during mental rotation only for the hand stimuli and no such direction-specific difference for the cube stimuli. The significant interaction between manual rotation direction and stimulus type supports this conclusion and reveals that this result is independent of the general difficulty level of the stimuli.

In a fourth experiment, we aimed to test directly whether the robust direction-specific interference effect was in fact specific for hand stimuli. Therefore, we directly compared the effects for hand stimuli with effects for other body and nonbody stimulus categories.

\section{EXPERIMENT 4 Stimulus Specificity of the Interference Effect}

\section{Method}

In the fourth experiment, the stimulus-specific interference effect found in the previous experiments was compared with the effects of further classes of stimuli in order to investigate the category specificity of our results.

Participants. The 22 right-handed participants (10 females and 12 males; mean age $=25.2$ years, $S D=3.5$, range $=21-29$ ) were psychology students at Frankfurt University who received course credit for their participation. None of the participants reported any health problems, and all had normal or corrected-to-normal vision. Participants were unaware of the purposes and predictions of the experiment until after test completion. 

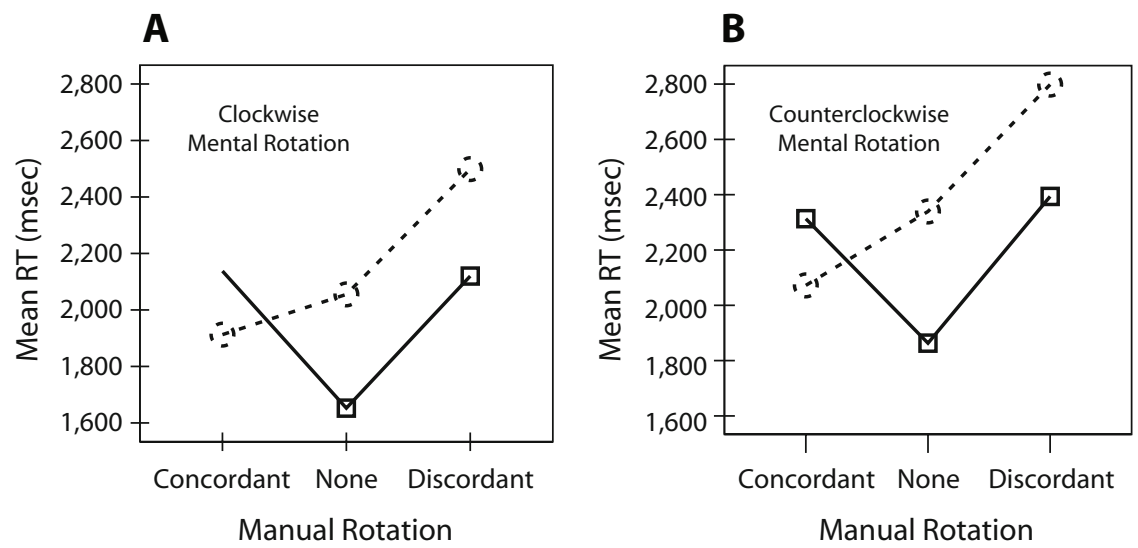

Figure 8. Interaction of the manual rotation effect with the two stimulus types (solid lines = cube figures; dotted lines $=$ hand figures), shown separately for clockwise mental rotation and counterclockwise mental rotation.

Stimuli. The stimuli consisted of grayscaled photographs showing hands, faces, or tools, as well as grayscaled figures of abstract cube stimuli. Two stimuli were either identical or mirror images across the vertical axis, and they were rotated two dimensionally at an angle of $40^{\circ}, 80^{\circ}$, or $120^{\circ}$ to the right. (For examples of the stimuli used, see Figure 9.) The order of the stimuli was randomized over the trials.

Apparatus and Procedure. The apparatus and procedure were identical to those used in the previous experiments. However, in this experiment, four different types of stimuli had to be mentally rotated: faces, hands, tools, and geometric figures.

The overall structure of the fourth experiment was a $3 \times 3 \times 4$ factorial design, with manual rotation direction (concordant vs. none vs. discordant), rotation of the stimuli $\left(40^{\circ}\right.$ vs. $80^{\circ}$ vs. $\left.120^{\circ}\right)$, and the four different stimulus types (faces vs. hands vs. tools vs. abstract figures) as within-participants factors.

The participants were asked to mentally rotate the stimuli in a clockwise direction while manually rotating the custom-made rotation device with the right hand. The manual rotation was either clockwise or counterclockwise at a speed of about $90^{\circ}$ per second, or the participants were instructed not to rotate. Participants had to decide whether the two presented objects were identical or mirror images and then had to register their decision by clicking the mouse as quickly and accurately as possible, using their left hand. The right mouse button (used by the left index finger) indicated that the object was a mirror image, and the left mouse button (operated by the left middle finger) indicated identical objects. Manual rotation speed and continuity were constantly monitored by the experimenter.

Data analysis. Prior to the inference statistical testing, we confirmed that no participant showed an error rate of $20 \%$ or higher. For the RT data, statistical outliers, defined as participants whose results differed more than two standard deviations from the mean, were identified and removed. Only trials with correct responses were considered and averaged for the different experimental conditions. The entire data set underwent a logarithmic transformation and was tested for normal distribution and variance homogeneity. These tests revealed the suitability of the RT data for parametric statistical testing. Tests of significance were performed using a three-factor repeated measures ANOVA with (1) rotational angle (three levels), (2) manual rotation direction (three levels), and (3) stimulus type (four levels) as the three repeated measures factors. On the basis of the results of this full-factorial ANOVA, several simple post hoc contrast analyses were performed for each condition.

\section{Results}

Stimulus type and angular distance effect. The three-factor ANOVA revealed significant main effects for stimulus type $[F(3,21)=28.13, p<.001]$ and angular rotation distance $[F(2,21)=28.90, p<.001]$. RTs were lowest for hand stimuli $(M=1,480 \mathrm{msec}, S D=444)$ and highest for face stimuli $(M=2,166 \mathrm{msec}, S D=642)$. Again, we found a clear angular distance effect with RTs increasing linearly from $40^{\circ}(M=2,217 \mathrm{msec}, S D=466)$, to $80^{\circ}(M=2,509 \mathrm{msec}, S D=552)$ to $120^{\circ}(M=2,660$, $S D=594)$ of mental rotation.

Manual rotation effect. In order to determine whether the interfering effect of manual rotation on mental rotation performance differed significantly among the four different stimulus categories, we tested for a significant interaction between the manual rotation direction factor (three levels) and the stimulus type factor (four levels). The full-factorial ANOVA model failed to reveal a significant interaction effect among all four levels of stimulus type and manual rotation direction $[F(6,21)=1.456, p=$ .209]. However, post hoc contrast analyses revealed that the interaction between stimulus type and manual rotation was significant when comparing only hand stimuli with cube figures $[F(2,21)=5.34, p=.022]$, whereas all other possible stimulus type $\times$ manual rotation direction comparisons revealed no significant interaction effects (see Figures 10C and 10D). For the abstract cubes, the RT was lowest without manual rotation $(M=2,322 \mathrm{msec}$, $S D=535)$ and significantly increased during both concordant $(M=2,677 \mathrm{msec}, S D=151)$ and discordant $(M=$ $2,589 \mathrm{msec}, S D=131)$ manual rotation, with no significant difference between rotation directions $[F(1,21)=$ $0.07, p=.80$; see Figure 10A].

The hand stimuli showed a different pattern of results in which the concordant manual rotation direction provided the lowest RT ( $M=1,806 \mathrm{msec}, S D=58)$, followed by the no hand movement condition $(M=1,935 \mathrm{msec}, S D=$ 95 ), with the discordant manual rotation yielding the slowest RT ( $M=2,179$ msec, $S D=139$; see Figure 10B). A post hoc simple contrast analysis revealed that only the RTs for the discordant manual rotation direction differed significantly, as compared with the no hand movement condition $[F(1,21)=4.81, p=.04]$ and the concordant 

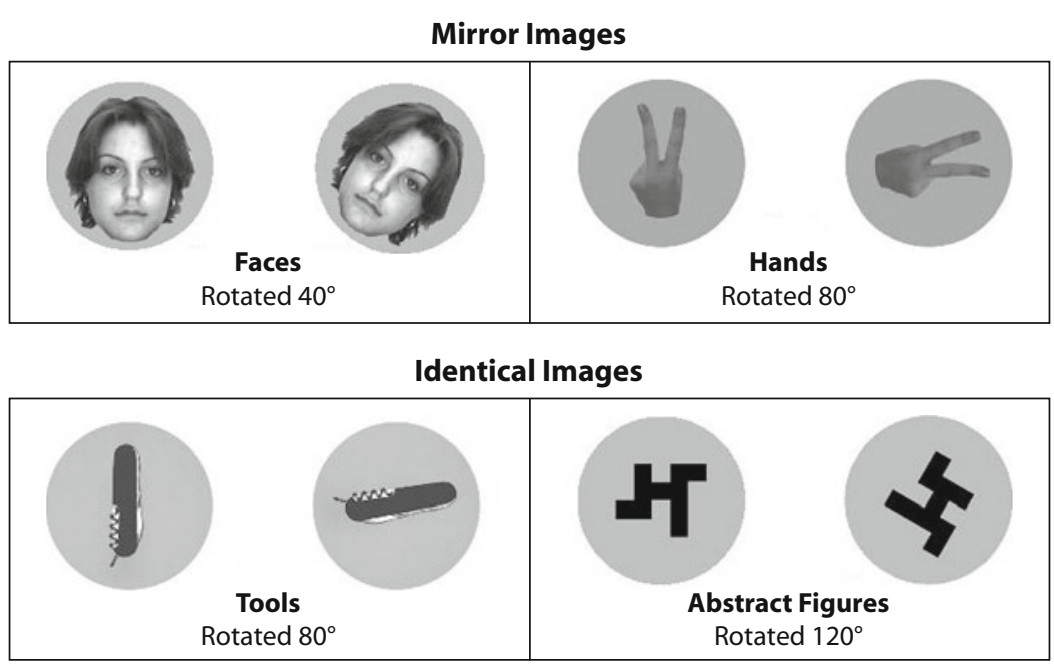

Figure 9. Illustrations of the mirror image condition of the face and hand stimuli (top) and the identical condition of the tools and abstract cube stimuli (bottom).

manual rotation direction condition $[F(1,21)=5.81$, $p=.03]$, whereas the contrast between the RTs for the concordant manual rotation and the no hand movement conditions failed to reach significance $[F(1,21)=0.79$, $p=.39]$. These results exactly replicate our findings of an object- and direction-specific interference between manual and mental rotation in Experiments 1-3.

\section{Summary}

This fourth experiment again clearly replicated the main findings of Experiments 1-3, which showed that the direction and the amount of interference between manual and mental rotation depended largely on the type of stimulus that had to be mentally rotated. Again, hand, but not cube, stimuli showed a direction-specific interference between manual and mental rotation. The mental rotation of tool or face stimuli, however, was not significantly affected by either concordant or discordant manual rotation.

\section{GENERAL DISCUSSION}

The four experiments presented in this study revealed three main results: First, interference between manual and mental rotation is influenced by the category of visual stimuli, with the main difference being between hands and geometric objects; second, the interference effect is independent of task difficulty; and third, the angular distance effect is present across all conditions and all different stimulus types. The absence of a significant interaction between the degree of rotational angle and the direction of manual rotation indicates that the interference is brought about by a shift in baseline performance, rather than by a systematic change in the rate of mental rotation.

Object-specific interference was found in all four experiments. The general pattern was that both concordant and discordant manual rotation disrupted mental rotation of cubes but only discordant manual rotation disrupted mental rotation of hands. We thus did not replicate the benefit for concordant versus discordant physical rotation on mental rotation of 3-D or 2-D geometric objects reported in previous studies (Wexler et al., 1998; Wohlschläger \& Wohlschläger, 1998). In the Wohlschläger and Wohlschläger study, both concordant and discordant rotation appeared to slow down mental rotation RTs, as compared with the RTs for the no-movement condition, at least for some rotational angles. However, Wohlschläger and Wohlschläger did not report a direct statistical comparison between the concordant or discordant rotation condition and the no-movement condition, and the Wexler et al. study did not have a no-movement condition at all. The point we are making, therefore, is that our finding that both concordant and discordant manual rotation slowed down the mental rotation of cubes does not seem to contradict previous findings. However, our failure to replicate the main finding of the previous studies, of a significant difference between the effects of concordant and discordant manual rotation on mental rotation of geometric objects, urges caution against the generalizability of this aspect of our findings.

One main difference between our paradigm and those of the studies mentioned is that we had both the target and the probe stimulus on the screen from the beginning of the trial, as in the classical Shepard and Metzler (1971) task, which meant that participants had to respond right away. In the Wohlschläger and Wohlschläger (1998) study, participants rotated their hands for $400 \mathrm{msec}$ before the probe stimulus appeared. They thus had $400 \mathrm{msec}$ to familiarize themselves with the target stimulus and become used to the manual rotation. In the Wexler et al. (1998) study, the participants had $5 \mathrm{sec}$ to familiarize themselves with the stimuli and prepare the rotation. Furthermore, Wexler et al. used the same 2-D object in each trial. We can thus assume that their participants were better prepared for their perceptual decisions than were the participants 

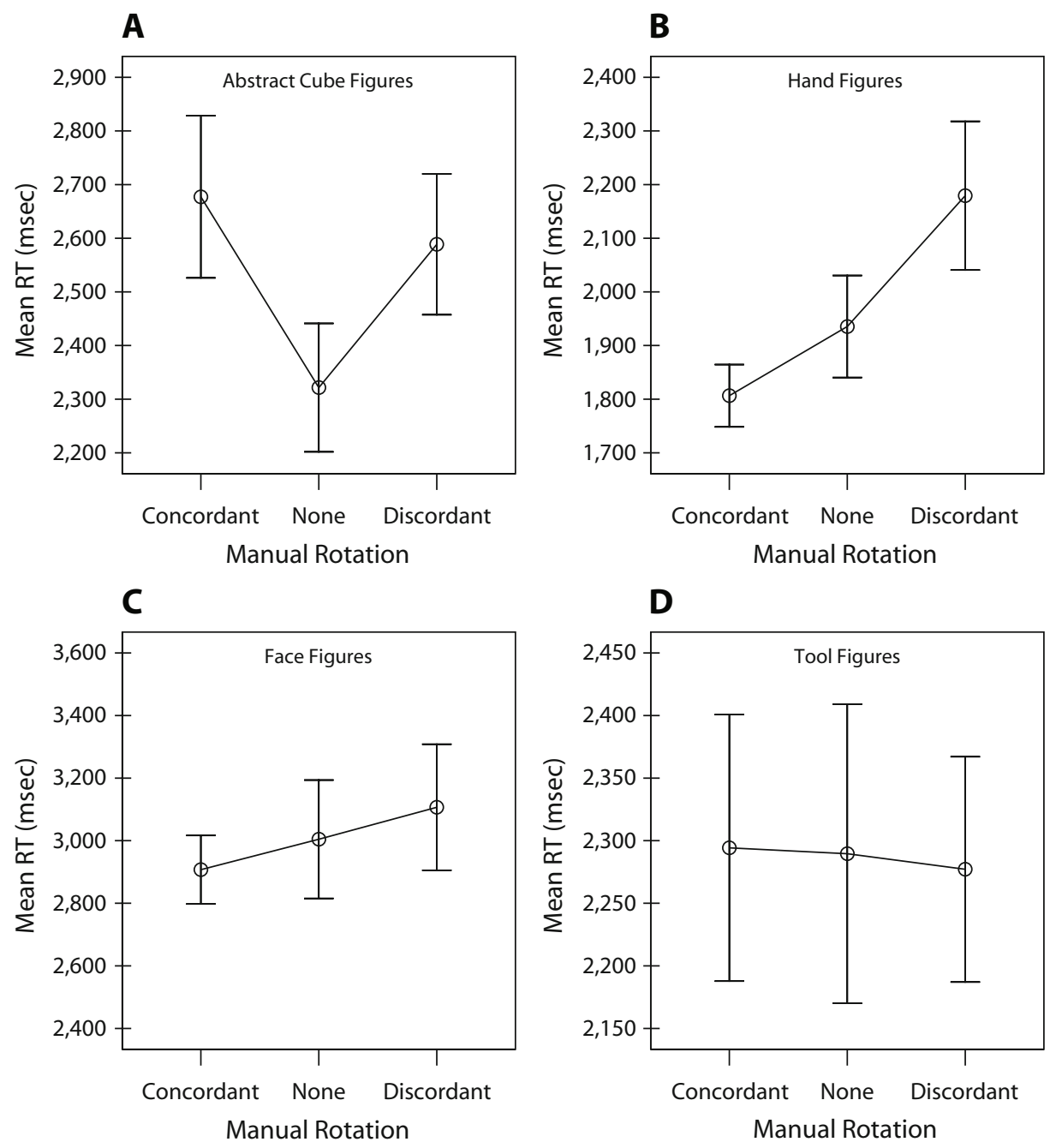

Figure 10. Mean reaction times (RTs) for the manual rotation direction in relation to the mental rotation direction (concordant direction, no hand movement, and discordant direction) for the four stimulus types: abstract cube, hand, face, and tool figures. Error bars represent standard errors of the means.

in our experiments, in which manual and mental rotation started concurrently. This may have protected the visual transformation system of Wexler et al.'s participants from some of the interference observed in the present studyspecifically, in the concordant condition. Another difference between the present and the two previous studies is that we required participants to rotate their wrists during the manual rotation condition, whereas the previous studies did not. Specificity of the interference for specific muscle groups seems unlikely at first, considering that most current explanations assume that the interference is due to conflicting representations at a higher level (Hommel, Müsseler, Aschersleben, \& Prinz, 2001). However, the motor or premotor representations of some muscle groups may be more susceptible to interaction with mental rotation or more densely connected with higher visual areas than are those of others. These concerns about the role of delay between target and probe and about the potential importance of specific muscle groups limit gen- eralizations from both our and previous findings. Future experiments that systematically vary these parameters should unravel the factors that determine the extent to which manual rotation inhibits or facilitates mental rotation of geometric objects.

However, the main finding of the present study is that the degree and pattern of disruption of mental rotation by manual rotation depends on the visual category, which we replicated across four experiments. Such a comparison of interference effects across categories had never been conducted before. The 3-D geometric objects (cubes) were affected by both directions of manual rotation, hands and hand-like objects (bundles of five carrots) were more affected by discordant rotation, and faces and tools were not affected at all. A number of theories might aid in the interpretation of the category-specific interference effect. Differences in mental rotation between body parts and other visual objects might be explained on the basis of two classes of task-specific transformation: object-based spa- 
tial transformation and egocentric perspective transformation (Zacks, Mires, Tversky, \& Hazeltine, 2000). With regard to this model, the cube figures could be assigned to an object-based spatial transformation, whereas the hand figures might be rotated on the basis of an egocentric perspective transformation. However, Zacks, Vettel, and Michelon (2003) also pointed out that object-based and perspective transformations are not mutually exclusive and that imagined movements of body parts have been shown to be relevant for a wide range of spatial transformation tasks.

Another model that might be adduced to interpret the present results derives from neuroimaging studies of the differential involvement of the motor cortex in mental rotation. These studies converge to suggest that the primary motor cortex is recruited only for mental rotation of hands, not for Shepard-Metzler-type objects (Kosslyn et al., 1998; Wraga, Thompson, Alpert, \& Kosslyn, 2003). This allows us to speculate that different activation in motor and premotor areas could account for our object-specific interference effect. The interference of both manual rotation directions during the mental rotation of the cube figures could result from the fact that both processes, the mental and the manual rotation, compete with each other for the same limited neuronal resources in premotor areas. The lack of interference of concordant manual rotation with hand stimuli, however, might be explained by involvement of the neurons of the primary motor cortex in both the actual rotation of the participant's hand and the mental rotation of the hand figures. In the case of the concordant rotation, these two processes would not compete. In the mental rotation task, the participants started to rotate the MRD immediately after stimulus presentation. They then identified the two hands, rotated one of them mentally, and then decided whether the hands were identical or mirrored. Thus, neurons of the primary motor cortex coding for the correct rotation direction were firing to support the manual rotation, which may have facilitated mental rotation in the same direction, offsetting any disruptive effect from competition at the premotor level.

The absence of interference of manual rotation with mental rotation of faces and tools, which seems striking at first in light of the strong and replicable interference effects for hands and cubes, may be explained by the special status of these visual categories. Unlike cubes or hands, faces and tools have their own specialized representations in the higher visual cortex, which also seem to support viewpoint-invariant perception (Jiang, Blanz, \& O'Toole, 2006; Weisberg, van Turennout, \& Martin, 2007). Mental rotation of these particular categories may therefore not interfere so much with the premotor network activated for both motor control and spatial transformation of cubes and hands.

The interference effect of the hand stimuli was stronger when participants rotated with their dominant right hand. This is in accordance with the results of Wohlschläger and Wohlschläger (1998), who also reported a stronger general interference effect when manually rotating with the dominant right hand. Furthermore, the imaging studies that reveal a specific involvement of the primary motor cortex during the mental rotation of hand stimuli often show a preponderance of this motor activity in the left hemisphere (Kosslyn et al., 1998; Wraga et al., 2003). Ganis et al. (2000) showed that the activation of the left primary motor cortex played a causal role in the mental rotation of pictures of hands as revealed by transcranial magnetic stimulation. On the basis of this left-hemispheric dominance of primary motor cortex activity during mental rotation tasks, one could expect the interference effect to be greater when the manual rotation is performed with the right hand than with the left. This assumption fits our data. However, the latter notion represents a post hoc interpretation of our empirical findings, and further research systematically investigating the hand dependence of this effect is needed.

Our finding that RTs increased linearly with increasing angles of rotation for all stimulus types conforms to the results of Shepard and Metzler (1971). This angular distance effect traditionally has been interpreted as strong evidence for the actual use of mental imagery by the participants (Kosslyn et al., 1998; Palmer, 1999; Vingerhoets, de Lange, Vandemaele, Deblaere, \& Achten, 2002). If an increment of time is required for each degree of angular disparity, participants can be assumed to perform such tasks by mentally rotating an object as if it were moving through the intermediate positions along a trajectory, as would occur if the object were physically rotated. Moreover, this linear effect of rotational angle on mental rotation time supports the view that mental images are picturelike representations. The analogue view of mental imagery (Kosslyn, 1994; Palmer, 1999) claims that if images are picture-like, their transformation should be continuous. Our results support this view, in that the linear effect of increasing time needed for the task with increasing angle appeared for all five stimulus types (cubes, carrots, faces, tools, and hand stimuli). This effect was also independent of the hand used for the manual rotation movement.

Recent neurophysiological and functional neuroimaging data, however, call into question the notion that angular distance effects are direct evidence for the use of mental imagery. Perrett, Oram, and Ashbridge (1998) proposed that object recognition is mediated by the accumulation of evidence in populations of viewpoint-specific neurons tuned to specific object features. Furthermore, Gauthier et al. (2002) dissociated the brain areas involved in viewpoint-dependent object recognition and mental rotation in an fMRI experiment using Shepard-and-Metzlerlike figures. Increasing viewpoint disparity in the mental rotation task led to higher activity in parietal areas that have also been implicated in motor planning (Georgopoulos, Lurito, Petrides, Schwartz, \& Massey, 1989). Conversely, higher activation in areas in the ventral temporal pathway (right inferior and middle temporal gyrus) was found for object recognition. Gauthier et al. concluded that the similar behavioral effects of viewpoint obtained in these two tasks were based on different neural substrates.

The interpretation of our results does not hinge on the analogue model of mental imagery, although our results 
are compatible with it. Likewise, the view that mental rotation is used to compensate for changes in viewpoint during object recognition, which is challenged by Gauthier et al. (2002), is not crucial to the interpretation of the present experiments. The only claim that we make on the basis of the present data is that the interference between manual and mental rotation, where present, is based on activation of shared neural resources for both types of rotation, a claim that is in fact supported by both Gauthier et al.'s and Georgopoulos et al.'s (1989) findings.

\section{CONCLUSION}

The divergent effects of concordant manual rotation on mental rotation of hand versus cube stimuli as revealed in this study seem to be plausible in light of the functional imaging and neurophysiology literature. This further supports the view, suggested by Wexler et al. (1998), that the motor system, instead of being a mere output device, may contribute to cognitive computations.

\section{AUTHOR NOTE}

A.T.S. was supported by Netherlands Organization for Scientific Research Grant 452-06-003. We thank Janine Bijsterbosch for help with data collection for Experiment 4. Correspondence concerning this article should be addressed to A. T. Sack, Maastricht University, Faculty of Psychology, Department of Cognitive Neuroscience, P. O. Box 616, 6200 MD Maastricht, The Netherlands (e-mail: a.sack@psychology.unimaas .nl) or D. E. J. Linden, School of Psychology, University of Wales, Bangor LL57 2AS, Wales (e-mail: d.linden@bangor.ac.uk).

\section{REFERENCES}

ANDERSON, J. R., \& Bower, G. H. (1973). Human associative memory. Washington, DC: Winston \& Sons.

Bonda, E., Petrides, M., Frey, S., \& Evans, A. (1995). Neural correlates of mental transformations of the body-in-space. Proceedings of the National Academy of Sciences, 92, 11180-11184.

Carpenter, P. A., Just, M. A., Keller, T. A., Eddy, W., \& ThulBORN, K. (1999). Graded functional activation in the visuospatial system with the amount of task demand. Journal of Cognitive Neuroscience, 11, 9-24.

Cohen, M. S., Kosslyn, S. M., Breiter, H. C., DiGirolamo, G. J., Thompson, W. L., Anderson, A. K., ET AL. (1996). Changes in cortical activity during mental rotation: A mapping study using functional MRI. Brain, 119, 89-100.

Ehrsson, H. H., Geyer, S., \& Naito, E. (2003). Imagery of voluntary movement of fingers, toes, and tongue activates corresponding body-part-specific motor representations. Journal of Neurophysiology, 90, 3304-3316.

Formisano, E., Linden, D. E. J., Di Salle, F., Trojano, L., EspoSITO, F., SACK, A. T., ET AL. (2002). Tracking the mind's image in the brain I: Time-resolved fMRI during visuospatial mental imagery. Neuron, 35, 185-194.

Ganis, G., Keenan, J. P., Kosslyn, S. M., \& Pascual-Leone, A. (2000). Transcranial magnetic stimulation of primary motor cortex affects mental rotation. Cerebral Cortex, 10, 175-180.

Gauthier, I., Hayward, W. G., Tarr, M. J., Anderson, A. W., SkudLARSKI, P., \& GORE, J. C. (2002). BOLD activity during mental rotation and viewpoint-dependent object recognition. Neuron, 34, 161-171.

Georgopoulos, A. P., Lurito, J. T., Petrides, M., Schwartz, A. B., \& MASSEY, J. T. (1989). Mental rotation of the neuronal population vector. Science, 243, 234-236.

Georgopoulos, A. P., \& Pellizzer, G. (1995). The mental and the neural: Psychological and neural studies of mental rotation and memory scanning. Neuropsychologia, 33, 1531-1547.

Goebel, R., Linden, D. E. J., Lanfermann, H., Zanella, F. E., \& SingER, W. (1998). Functional imaging of mirror and inverse read- ing reveals separate coactivated networks for oculomotion and spatial transformations. NeuroReport, 9, 713-719.

Goldstein, E. B. (2002). Sensation and perception (6th ed.). Belmont, CA: Wadsworth.

Hommel, B., Müsseler, J., Aschersleben, G., \& Prinz, W. (2001). The Theory of Event Coding (TEC): A framework for perception and action planning. Behavioral \& Brain Sciences, 24, 849-878.

JAMES, W. (1890). The principles of psychology (Vols. 1 and 2). New York: Holt

Jiang, F., Blanz, V., \& O'Toole, A. J. (2006). Probing the visual representation of faces with adaptation: A view from the other side of the mean. Psychological Science, 17, 493-500.

KossLyn, S. M. (1980). Image and mind. Cambridge, MA: Harvard University Press.

KossLYN, S. M. (1994). Image and brain: The resolution of the imagery debate. Cambridge, MA: MIT Press.

Kosslyn, S. M., Behrmann, M., \& Jeannerod, M. (1995). The cognitive neuroscience of mental imagery. Neuropsychologia, 33, 13351344.

Kosslyn, S. M., Digirolamo, G. J., Thompson, W. L., \& Alpert, N. M. (1998). Mental rotation of objects versus hands: Neural mechanisms revealed by positron emission tomography. Psychophysiology, 35, 151-161.

KossLyn, S. M., \& Thompson, W. L. (2003). When is early visual cortex activated during visual mental imagery? Psychological Bulletin, 129, 723-746.

Lamm, C., Windischberger, C., Leodolter, U., Moser, E., \& BAUER, H. (2001). Evidence for premotor cortex activity during dynamic visuospatial imagery from single-trial functional magnetic resonance imaging and event-related slow cortical potentials. NeuroImage, 14, 268-283.

PAIVIO, A. (1971). Imagery and verbal processes. New York: Holt, Rinehart \& Winston.

Palmer, S. E. (1999). Vision science. Cambridge, MA: MIT Press.

PARsons, L. M. (1987). Imagined spatial transformation of one's body. Journal of Experimental Psychology: General, 116, 172-191.

Parsons, L. M., Fox, P. T., Downs, J. H., Glass, T., Hirsch, T. B., Martin, C. C., ET AL. (1995). Use of implicit motor imagery for visual shape discrimination as revealed by PET. Nature, 375, 54-57.

Perrett, D. I., Oram, M. W., \& Ashbridge, E. (1998). Evidence accumulation in cell populations responsive to faces: An account of generalisation of recognition without mental transformations. Cognition, 67, 111-145.

PyLyshyn, Z. W. (1973). What the mind's eye tells the mind's brain: A critique of mental imagery. Psychological Bulletin, 80, 1-24.

Richardson, J. T. E. (1999). Imagery. Hove, U.K.: Psychology Press.

Richter, W., Somorjai, R., Summers, R., Jarmasz, M., Menon, R. S., GATIS, J. S., ET AL. (2000). Motor area activity during mental rotation studied by time-resolved single-trial fMRI. Journal of Cognitive Neuroscience, 12, 310-320.

Riesenhuber, M., \& Poggio, T. (2000). Models of object recognition. Nature Neuroscience, 3(Suppl.), 1199-1204.

Sack, A. T., Sperling, J., Prvulovic, D., Formisano, E., Goebel, R., Di SALLE, F., ET AL. (2002). Tracking the mind's image in the brain II: Transcranial magnetic stimulation reveals parietal asymmetry in visuospatial imagery. Neuron, 35, 195-204.

SeKiYAMA, K. (1982). Kinesthetic aspects of mental representations in the identification of left and right hands. Perception \& Psychophysics, 32, 89-95.

Sekiyama, K., Miyauchi, S., Imaruoka, T., Egusa, H., \& Tashiro, T. (2000). Body image as a visuomotor transformation device revealed in adaptation to reversed vision. Nature, 407, 374-377.

Shepard, R. N., \& Metzler, J. (1971). Mental rotation of threedimensional objects. Science, 171, 701-703.

TARR, M. J. (1999). Mental rotation. In R. A. Wilson \& F. C. Keil (Eds.), The MIT encyclopedia of the cognitive sciences (pp. 531-533). Cambridge, MA: MIT Press.

Vingerhoets, G., De Lange, F .P., Vandemaele, P., Deblaere, K., \& ACHTEN, E. (2002). Motor imagery in mental rotation: An fMRI study. NeuroImage, 17, 1623-1633.

Weisberg, J., van Turennout, M., \& Martin, A. (2007). A neural system for learning about object function. Cerebral Cortex, 17, 513521. 
Wexler, M., Kosslyn, S. M., \& Berthoz, A. (1998). Motor processes in mental rotation. Cognition, 68, 77-94.

WoHLSCHLÄGER, A. (2001). Mental object rotation and the planning of hand movements. Perception \& Psychophysics, 63, 709-718.

WohlschläGER, A., \& WoHLSCHLÄGER, A. (1998). Mental and manual rotation. Journal of Experimental Psychology: Human Perception \& Performance, 24, 397-412.

Wraga, M., Thompson, W. L., Alpert, N. M., \& Kosslyn, S. M. (2003). Implicit transfer of motor strategies in mental rotation. Brain \& Cognition, 52, 135-143.
Zacks, J. M., Mires, J., Tversky, B., \& Hazeltine, E. (2000). Mental spatial transformations of objects and perspective. Spatial Cognition \& Computation, 2, 315-332.

Zacks, J. M., Vettel, J. M., \& Michelon, P. (2003). Imagined viewer and object rotations dissociated with event-related fMRI. Journal of Cognitive Neuroscience, 15, 1002-1018.

(Manuscript received January 26, 2005; revision accepted for publication June 5, 2007.) 